\title{
A program of nurse algorithm-guided care for adult patients with acute minor illnesses in primary care
}

Núria Fabrellas ${ }^{1 *}$, Carmen Sánchez ${ }^{2}$, Eulàlia Juvé ${ }^{1,3}$, Eva Aurin $^{3}$, Dolors Monserrat $^{4}$, Esther Casanovas $^{5}$ and Magali Urrea $^{2}$

\begin{abstract}
Background: Attention to patients with acute minor-illnesses requesting same-day consultation represents a major burden in primary care. The workload is assumed by general practitioners in many countries. A number of reports suggest that care to these patients may be provided, at in least in part, by nurses. However, there is scarce information with respect to the applicability of a program of nurse management for adult patients with acute minor-illnesses in large areas. The aim of this study is to assess the effectiveness of a program of nurse algorithm-guided care for adult patients with acute minor illnesses requesting same-day consultation in primary care in a largely populated area.
\end{abstract}

Methods: A cross-sectional study of all adult patients seeking same day consultation for 16 common acute minor illnesses in a large geographical area with 284 primary care practices. Patients were included in a program of nurse case management using management algorithms. The main outcome measure was case resolution, defined as completion of the algorithm by the nurse without need of referral of the patient to the general practitioner. The secondary outcome measure was return to consultation, defined as requirement of new consultation for the same reason as the first one, in primary care within a 7-day period.

Results: During a two year period (April 2009-April 2011), a total of 1,209,669 consultations were performed in the program. Case resolution was achieved by nurses in $62.5 \%$ of consultations. The remaining cases were referred to a general practitioner. Resolution rates ranged from $94.2 \%$ in patients with burns to $42 \%$ in patients with upper respiratory symptoms. None of the 16 minor illnesses had a resolution rate below $40 \%$. Return to consultation during a 7-day period was low, only 4.6\%.

Conclusions: A program of algorithms-guided care is effective for nurse case management of patients requesting same day consultation for minor illnesses in primary care.

Keywords: Nurse case management, Acute diseases/nursing, Nurse practitioner, Primary health care

\section{Background}

In the current context of rapidly growing healthcare demands there is generalized interest for an increasing role of nurses in primary care [1]. The USA Institute of Medicine has called for broadening nurses' scope of practice in primary care [2]. Likewise, in the UK, the government has issued a policy that expands the role of nurses in the community practice [3]. Management of patients with acute

\footnotetext{
* Correspondence: nfabrellas@ub.edu

'School of Nursing, University of Barcelona, Institut d'Investigació Biomèdica de Bellvitge (IDIBELL), Campus de Ciències de la Salut Bellvitge, Feixa Llarga s/n Pavello de Govern, 3a planta, 08907, L'Hospitalet de Llobregat, Catalunya, Spain Full list of author information is available at the end of the article
}

minor illnesses is an exemplary case to be considered. In many countries, the healthcare to these patients is delivered on a same day basis (that is, the same day the consultation occurs) by general practitioners (GPs) that have to find a spot in their busy agendas to visit these patients. There is a large body of evidence from randomized studies and systematic reviews indicating that nurses can provide these patients with care of similar quality to that provided by GPs with a similar or even better patient satisfaction [4-11]. However, despite this available evidence, care of patients with acute minor illnesses is still, by and large,

\section{Biomed Central}


delivered by GPs in most countries with little or no role for nurses.

The main problems preventing an expansion of the role of nurses in primary care are the disparity in the levels of nursing education in different countries and some regulatory barriers, particularly drug prescription. In Spain, the education of registered nurses $(\mathrm{RN})$ is at the graduate level but they can only prescribe certain drugs under the supervision of the GP. In order to expand the role of RN and still adhering with the legislation, the Catalonian Institute of Health developed a program of nurse care for patients with acute minor illnesses that uses management algorithms, which are included in the computerized health record system. This study assessed the effectiveness of this program over a two-year period in a highly populated area with approximately 6 million inhabitants.

\section{Methods}

\section{Design of the study}

This is a cross-sectional study with retrospective analysis of data from patients attended in a program of nurse algorithm-guided care for adults with acute minor illnesses in primary care. Data were collected prospectively in the electronic medical record at the time of patient consultation.

\section{Study population}

Adult patients requesting consultation without appointment for 16 different acute minor illnesses over a two year period (April 2009-April 2011) were included in the study. The analyzed geographical area includes 284 primary care practices attending a population of approximately 6 million inhabitants in Catalonia, northeast of Spain. Consecutive sampling technique was used to include cases in the study. All subjects older than 14 years requesting same day consultation for any of 16 acute minor illnesses (see later) were included in the study.

\section{Program of nurse algorithm-guided care for patients with acute minor illnesses}

The program was made by a group of expert nurses and GPs and consisted of a general protocol and 16 management algorithms of very common acute minor illnesses in adults seen in primary care [12]. The 16 acute minor illnesses included in the program were the following: skin injury, upper respiratory symptoms, sore throat, lower urinary symptoms, acute diarrhea, low back pain, increased arterial pressure, pink eye, burns, tooth pain, twisted ankle, emergency contraception, anxiety attacks, skin fold dermatitis, flu, and nose bleeding. Management algorithms were made following the most recent available evidence for each of the 16 conditions. Training sessions were performed in all practices before the implementation of the program. Once the program started, all patients seeking same day consultation were initially seen by a nurse. In all practices, there was one nurse dealing with these patients 5 days per week. All nurses of each practice participated in the program in turns. The general protocol included the following steps: 1) assessment of main signs and symptoms; 2) recognition of previous health problems and their treatments; 3 ) identification of the main reason for consultation. If the patient's condition was included in one of the 16 minor illnesses the corresponding predefined management algorithm was applied. Algorithms included signs of alarm to identify patients with a potentially severe condition requiring consultation with a GP. If signs of alarm were not present, the nurse completed the algorithm that in some cases required drug prescription. If signs of alarm were detected, the nurse referred the patient to the GP, who was always available for a same day visit. The protocol and management algorithms were included in the computerized medical system so that all steps could be followed easily at the time of writing in patients' electronic medical records. As an example, the management algorithms of the 4 most common minor illnesses are shown in Tables 1 , 2,3 and 4 .

\section{Data collection and analysis}

Data from all consecutive patients seen in the program during the 2-yr period of the study were downloaded

Table 1 Nurse treatment protocol for patients with skin injury

\begin{tabular}{|c|c|}
\hline \multirow{3}{*}{ Signs of alarm } & $\begin{array}{l}\text { Involvement of head, joints, nerves } \\
\text { and/or tendons }\end{array}$ \\
\hline & Profuse hemorrhage \\
\hline & Report to the police, if violence was involved \\
\hline \multirow{15}{*}{ Treatment approach } & No signs of infection or bites \\
\hline & 1. Cleansing and disinfection with clorhexidine \\
\hline & 2. Moist environment \\
\hline & $\begin{array}{l}\text { 3. Suture, if needed (open injuries of } \\
\text { less than } 6 \text { hours). }\end{array}$ \\
\hline & 4. Haemostasis, if needed. \\
\hline & 5. If pain, paracetamol $500 \mathrm{mg}$ tid, as needed \\
\hline & 6. Tetanus prophylaxis, if needed. \\
\hline & Signs of infection or bite injury \\
\hline & 1. Cleansing and disinfection with clorhexidine. \\
\hline & 2. Moist environment \\
\hline & 3. No suture \\
\hline & 4. Haemostasis, if needed \\
\hline & $\begin{array}{l}\text { 5. If pain, paracetamol 500/1,000 mg qid, } \\
\text { as needed. }\end{array}$ \\
\hline & $\begin{array}{l}\text { 6. Tetanus prophylaxis, if needed. In bites, } \\
\text { consider rabies prophylaxis }\end{array}$ \\
\hline & $\begin{array}{l}\text { 7. Amoxycillin-clavulanic acid } 500 / 125 \mathrm{mg} \text { tid } \\
\text { for } 8 \text { days. Erithromycin } 500 \mathrm{mg} \text { qid for } 8 \text { days } \\
\text { in patients with known allergy to penicillin. }\end{array}$ \\
\hline
\end{tabular}


Table 2 Nurse treatment protocols for patients with upper respiratory symptoms

\begin{tabular}{|c|c|}
\hline \multirow{15}{*}{ Signs of alarm } & $\begin{array}{l}\text { Asthma or chronic pulmonary } \\
\text { obstructive disease }\end{array}$ \\
\hline & Shortness of breath \\
\hline & Immunosuppressive therapy \\
\hline & Temperature $>40^{\circ} \mathrm{C}$ \\
\hline & Fever for more than 3 days \\
\hline & Symptoms for more than 14 days \\
\hline & Abnormal lung auscultation \\
\hline & Pregnancy or lactation \\
\hline & Decompensated cardiac disease \\
\hline & Diabetes mellitus not well controlled \\
\hline & Recent hospitalization \\
\hline & Severe ear pain \\
\hline & Severe headache \\
\hline & Chest pain \\
\hline & Severe weakness \\
\hline \multirow[b]{2}{*}{ Treatment approach } & $\begin{array}{l}\text { 1. In patients with runny nose, cough with } \\
\text { sputum, fatigue and/or body aches, } \\
\text { paracetamol } 500 / 1,000 \mathrm{mg} / \text { day tid or qid, } \\
\text { plus frequent fluid intake. }\end{array}$ \\
\hline & $\begin{array}{l}\text { 2. In patients with cough without sputum, } \\
\text { dextrometorphan } 15-30 \mathrm{mg} \text { tid or qid or } \\
\text { codeine } 10-20 \mathrm{mg} \text { every } 4-5 \text { hours } \\
\text { (if patient does not take monoamino } \\
\text { oxidase inhibitors). }\end{array}$ \\
\hline
\end{tabular}

directly from the computerized electronic health record system using specific software and incorporated into a database for analysis.

The main outcome measure of the study was case resolution, defined as completion of the management algorithm without the need of referral of the patient to the GP. The secondary outcome measure was return to consultation, defined as requirement of new consultation for the same reason as the first one in primary care, either with a nurse or GP, within a 7-day period. Comparison of frequencies of the different outcomes was performed with the chi-square test. The analysis was performed using the SPSS 15 for Windows (SPSS Inc, Chicago, IL).

\section{Ethical approval}

The study was approved by the Institut d'Investigació en Atenció Primària (IDIAP Jordi Gol) and followed the current regulations for data confidentiality.

\section{Results}

During a two-year period, more than $1,200,000$ same-day consultations corresponding to 16 predefined acute minor illnesses in adults were performed by nurses under the current program using management algorithms in primary care. This corresponds to an average of approximately
Table 3 Nurse treatment protocol for patients with sore throat

\begin{tabular}{ll}
\hline Symptoms for more than 7 days \\
\hline Temperature $>40^{\circ} \mathrm{C}$ or $>38^{\circ} \mathrm{C}$ for \\
more than 3 days
\end{tabular}

50,000 consultations per month and 2,400 consultations per working day.

The 16 minor illnesses and their corresponding frequencies are shown in Table 5. The most common were skin injury, upper respiratory symptoms, sore throat, lower urinary symptoms, acute diarrhea, and low back pain. Each of these 6 minor illnesses had a frequency greater than 5\% (range 23.6 to 6.3) and all 6 together accounted for more than three quarters of all consultations (77.5\%). The less common minor illnesses were nose bleeding, flu, skin fold dermatitis, and anxiety attacks, with a frequency lower than $2 \%$ each. The remaining conditions, increased arterial pressure, pink eye, burns, tooth pain, twisted ankle, and emergency contraception, had individual frequencies between 2 and $5 \%$. In the second year the number of consultations increased by $10.3 \%$ with respect to the first year (Table 5).

Overall, case resolution was achieved in a high proportion of cases (62.5\%). The remaining $37.5 \%$ of cases were 
Table 4 Nurse protocol for patients with lower urinary symptoms

\begin{tabular}{|c|c|}
\hline \multirow{16}{*}{ Signs of alarm } & Symptoms for more than 7 days \\
\hline & Fever and/or chills \\
\hline & Pregnancy or lactation \\
\hline & Male sex \\
\hline & Age greater than 65 \\
\hline & Diabetes mellitus \\
\hline & $\begin{array}{l}\text { Recurrent urinary tract infection ( } \geq 2 \text { infections } \\
\text { within a } 6 \text {-month period) }\end{array}$ \\
\hline & Recent urinary tract infection (less than 2 weeks) \\
\hline & Pyelonephritis within one year period \\
\hline & Bladder catheter \\
\hline & Vaginal discharge \\
\hline & Nausea and/or vomiting \\
\hline & Abdominal pain \\
\hline & Known abnormalities in urinary tract \\
\hline & Gross hematuria \\
\hline & Immunosuppressive therapy \\
\hline \multirow{3}{*}{ Treatment approach } & $\begin{array}{l}\text { 1. Amoxycillin-clavulanic acid } 500 / 125 \mathrm{mg} \\
\text { tid for } 5 \text { days or norfloxacin } 400 \mathrm{mg} \text { bid for } \\
3 \text { days. Norfloxacin prescribed to patients } \\
\text { with known allergy to penicillin. }\end{array}$ \\
\hline & $\begin{array}{l}\text { 2. Hygiene recommendations: cleanliness of } \\
\text { the genital area, frequent bladder voiding, } \\
\text { cotton-crotch underwear. }\end{array}$ \\
\hline & $\begin{array}{l}\text { 3. Return to consultation if signs of alarm } \\
\text { develop (chills/fever, red urine, } \\
\text { abdominal or lumbar pain...) }\end{array}$ \\
\hline
\end{tabular}

not solved by the nurse and had to be referred to the GP. The frequency of case resolution over the two-year period varied widely among the different minor illnesses (Table 6). Resolution rates were very high (over 90\%) in conditions such as burns, emergency contraception, or skin injury. In other minor illnesses, such as skin fold dermatitis, increased arterial pressure, twisted ankle, nose bleeding, acute diarrhea, tooth pain, anxiety attacks, and low back pain, resolution rates ranged between 50 and $70 \%$. The lowest resolution rates (below 50\%) were found in upper respiratory symptoms, pink eye, lower urinary symptoms, sore throat, and flu, which ranged between $42.0 \%$ and $49.9 \%(\mathrm{p}<0.001)$. Nonetheless, it is important to note that none of the 16 different minor illnesses had a resolution rate below $40 \%$ (Table 6). The overall resolution rate observed during the second year was significantly higher than that of the first year $(63.1 \%$ vs 61.8 , respectively, $\mathrm{p}<0.0001$ ) (Table 6). When rates of resolution between the first and second year were compared for each of the 16 minor illnesses, it was found that resolution rates increased significantly in 8 (burns, emergency contraception, acute diarrhea, tooth pain, sore throat, lower urinary symptoms, pink eye and upper respiratory symptoms), decreased significantly in 5 , and did not change significantly
Table 5 Frequency of the $\mathbf{1 6}$ minor illnesses during the 2-year period

\begin{tabular}{lccc}
\hline & \multicolumn{2}{c}{ Frequency } & \\
\cline { 2 - 3 } Category & First year & Second year & Total \\
\hline Skin injury & $130,988(22.8)$ & $154,924(24.4)$ & $285,912(23.6)$ \\
Upper respiratory & $118,470(20.6)$ & $109,293(17.2)$ & $227,763(18.8)$ \\
symptoms & & & \\
Sore throat & $67,550(11.7)$ & $71,386(11.3)$ & $138,936(11.5)$ \\
Lower urinary symptoms & $49,996(8.7)$ & $69,126(10.9)$ & $119,122(9.9)$ \\
Acute diarrhea & $42,431(7.4)$ & $47,708(7.5)$ & $90,139(7.5)$ \\
Low back pain & $35,059(6.1)$ & $41,033(6.5)$ & $76,092(6.3)$ \\
Increased arterial pressure & $23,632(4.1)$ & $29,344(4.6)$ & $52,976(4.4)$ \\
Pink eye & $21,485(3.7)$ & $24,946(3.9)$ & $46,431(3.8)$ \\
Burns & $18,540(3.2)$ & $19,468(3.1)$ & $38,008(3.1)$ \\
Tooth pain & $16,302(2.8)$ & $19,319(3.0)$ & $35,621(2.9)$ \\
Twisted ankle & $12,789(2.2)$ & $13,427(2.5)$ & $26,216(2.2)$ \\
Emergency contraception & $13,652(2.4)$ & $10,974(1.7)$ & $24,626(2.0)$ \\
Anxiety attacks & $10,125(1.8)$ & $11,720(1.8)$ & $21,845(1.8)$ \\
Skin fold dermatitis & $5,794(1.0)$ & $5,676(0.9)$ & $11,470(1.0)$ \\
Flu & $5,927(1.0)$ & $3,635(0.6)$ & $9,562(0.8)$ \\
Nose bleeding & $2,448(0.4)$ & $2,502(0.4)$ & $4,950(0.4)$ \\
Total & $\mathbf{5 7 5 , 1 8 9}$ & $\mathbf{6 3 4 , 4 8 0}$ & $\mathbf{1 , 2 0 9 , 6 6 9}$ \\
\hline
\end{tabular}

Numbers in brackets are percentages of the total number of patients during each period.

Table 6 Resolution rates of the 16 acute minor illnesess during the first and second year of the program

\begin{tabular}{|c|c|c|c|c|}
\hline \multirow[b]{2}{*}{ Category } & \multicolumn{3}{|c|}{ Resolution (\%) } & \multirow[b]{2}{*}{$\mathbf{p}^{*}$} \\
\hline & First year & Second year & Total & \\
\hline Burns & 93.5 & 94.8 & 94.2 & $<0.0001$ \\
\hline Emergency contraception & 90.8 & 92.8 & 91.7 & $<0.0001$ \\
\hline Skin injury & 91.6 & 90.9 & 91.2 & $<0.0001$ \\
\hline Skin fold dermatitis & 71.3 & 69.3 & 70.3 & 0.017 \\
\hline Increased arterial pressure & 70.0 & 67.2 & 68.4 & $<0.0001$ \\
\hline Twisted ankle & 66.0 & 66.6 & 66.3 & NS \\
\hline Nose bleeding & 64.1 & 65.1 & 64.6 & NS \\
\hline Acute diarrhea & 62.1 & 63.5 & 62.8 & $<0.0001$ \\
\hline Tooth pain & 54.1 & 56.1 & 55.2 & $<0.0001$ \\
\hline Anxiety attacks & 55.3 & 53.1 & 54.1 & 0.0013 \\
\hline Low back pain & 51.4 & 51.2 & 51.6 & NS \\
\hline Flu & 51.1 & 47.9 & 49.9 & 0.0026 \\
\hline Sore throat & 45.7 & 49.9 & 47.8 & $<0.0001$ \\
\hline Lower urinary symptoms & 46.8 & 47.5 & 47.2 & 0.018 \\
\hline Pink eye & 45.5 & 46.7 & 46.1 & 0.009 \\
\hline Upper respiratory symptoms & 41.4 & 42.7 & 42 & $<0.0001$ \\
\hline Total & 61.8 & 63.1 & 62.5 & $<0.0001$ \\
\hline
\end{tabular}

*Comparison between the first and second year rates. 
in the remaining 3 (Table 6). When patients were categorized in two groups according to age (using an arbitrary cutoff of 50 years), there were significant differences in the resolution rates of some categories; however, the overall resolution rate was identical in the two groups, indicating that age by itself has no major impact on the resolution rate in a program of nurse algorithm-guided care for adult patients (Table 7).

In the whole series, the proportion of patients who returned to consultation after the nurse visit was low and averaged only $4.6 \%$. The highest return rate was observed in skin injury and the lowest in emergency contraception ( $8.6 \%$ and $0.4 \%$, respectively) (Table 8 ). The return to consultation of all 16 minor illnesses together was significantly higher during the second year compared to that of the first year $(5.1 \%$ vs $4.2 \%, \mathrm{p}<0.0001)$ (Table 8$)$. However, this increase in the return to consultation was basically due to the increment in burns and skin injury rates.

\section{Discussion}

In the current study we report the results of a program of nurse algorithm-guided care for adult patients with acute minor illnesses in primary care in a very large cohort of patients $(1,209,669$ consultations) over a two-

Table 7 Comparison of resolution rate of the different 16 minor acute illness in patients included categorized according to age*

\begin{tabular}{|c|c|c|c|}
\hline \multirow[b]{2}{*}{ Category } & \multicolumn{3}{|c|}{ Resolution by age (\%) } \\
\hline & $\begin{array}{c}\leq 50 \\
(n=751,325)\end{array}$ & $\begin{array}{c}>50 \\
(n=458,344)\end{array}$ & $\mathrm{p}$ \\
\hline Burns & 94.0 & 94.7 & NS \\
\hline $\begin{array}{l}\text { Emergency } \\
\text { contraception }\end{array}$ & - & - & - \\
\hline Skin injury & 89.7 & 92.0 & $<0.0001$ \\
\hline Skin fold dermatitis & 67.6 & 70.4 & NS \\
\hline Increased arterial pressure & 61.1 & 68.7 & $<0.0001$ \\
\hline Twisted ankle & 65.6 & 65 & NS \\
\hline Nose bleeding & 68.6 & 60.1 & 0.0046 \\
\hline Acute diarrhea & 64.5 & 55.4 & $<0.0001$ \\
\hline Tooth pain & 54.4 & 57.1 & 0.0221 \\
\hline Anxiety attacks & 50.3 & 60.3 & $<0.0001$ \\
\hline Low back pain & 49.7 & 52.5 & $<0.0001$ \\
\hline Flu & 50.7 & 45.3 & 0.0048 \\
\hline Sore throat & 48.5 & 49.3 & NS \\
\hline Lower urinary symptoms & 47.0 & 45.9 & $<0.0001$ \\
\hline Pink eye & 45.3 & 47.2 & 0.0103 \\
\hline $\begin{array}{l}\text { Upper respiratory } \\
\text { symptoms }\end{array}$ & 44.8 & 37.4 & $<0.0001$ \\
\hline Total & 60.1 & 60.1 & \\
\hline
\end{tabular}

*Emergency contraception was not included in the analysis because number of patients in the $>50$ group was negligible.
Table 8 Return to consultation of the $\mathbf{1 6}$ acute minor illness during the first and second year of the program

\begin{tabular}{lcccl}
\hline & \multicolumn{3}{c}{ Return to consultation (\%) } & \\
\cline { 2 - 4 } Category & First year & Second year & Total & p $^{*}$ \\
\hline Burns & 7.2 & 9.3 & 8.3 & $<0.0001$ \\
Emergency contraception & 0.4 & 0.3 & 0.4 & NS \\
Skin injury & 7.5 & 9.6 & 8.6 & $<0.0001$ \\
Skin fold dermatitis & 1.4 & 0.5 & 1.0 & $<0.0001$ \\
Increased arterial pressure & 2.4 & 3.0 & 4.9 & 0.0006 \\
Twisted ankle & 1.5 & 1.9 & 1.7 & 0.0182 \\
Nose bleeding & 4.0 & 3.5 & 3.8 & NS \\
Acute diarrhea & 1.3 & 1.0 & 1.1 & 0.008 \\
Tooth pain & 1.9 & 2.1 & 2.0 & NS \\
Anxiety attacks & 1.4 & 1.1 & 1.3 & NS \\
Low back pain & 5.4 & 4.7 & 5.0 & 0.0008 \\
Flu & 1.0 & 0.7 & 0.8 & NS \\
Sore throat & 1.3 & 1.1 & 1.2 & 0.0239 \\
Lower urinary symptoms & 1.5 & 1.7 & 1.6 & NS \\
Pink eye & 1.3 & 1.3 & 1.3 & NS \\
Upper respiratory symptoms & 1.9 & 1.7 & 1.8 & 0.0063 \\
Total & $\mathbf{4 . 2}$ & $\mathbf{5 . 1}$ & $\mathbf{4 . 6}$ & $<0.0001$ \\
\hline *Comparison between the first and second year rates. & &
\end{tabular}

year period. The program was designed to fulfill two criteria: treatment algorithms and close collaboration between nurses and GPs. The program was also intended to solve some of the concerns raised about the effectiveness and appropriateness of extending the role of nurses to same day consultations for acute minor illnesses in primary care, thus substituting GPs in this function. One of these concerns is that nurses may work independently of GPs which may result in lack of consultation in the case of uncertainty [13]. To avoid this potential effect, the program reported herein was the result of a collective effort between GPs and nurses. Moreover, in all management algorithms a number of signs of alarm were included, so that patients had to be sent to the GP for an urgent consultation if one of the signs of alarm was present. Another concern is that patients, at least in some countries, are not convinced that nurses have the sufficient education and knowledge to deal with these type of problems compared to GPs [11-14]. The close cooperation between nurses and GPs put forward in our program may help convince patients that nurses have the sufficient expertise to solve the problems in the majority of cases and that a consultation with the GP will be requested in case of uncertainty. A major issue that has limited the applicability of programs of nurse same day consultation for patients with acute minor illnesses in many countries has been the fact that nurses cannot prescribe certain drugs. On the other hand, nurses may 
have the feeling of a lack of appropriate pharmacological knowledge together with insufficient confidence in this area. The use of management algorithms allows restricting the drug prescription to certain conditions, following evidence-based guidelines. Moreover, the use of management algorithms may have the additional benefits of improving the adherence to protocols and reducing inter-professional variability in their application. Finally, the incorporation of the management algorithms in the computerized health record system is essential for their accurate application and also allows for a periodical assessment of the efficacy of the program.

The program reported here has some similarities with the walk-in centers from United Kingdom [15]. These are centers specifically created to provide care to patients with minor illnesses (minor injuries in many instances), which are usually run by nurses. However, there are significant differences that should be mentioned. First, our program was run in general practices and not in new and specific centers. Second, the program was devoted to a large number of acute minor illnesses that represent an important workload in primary care. Third, although the program was run by nurses, there was a close cooperation between nurses and GPs, which allowed the resolution of complex cases. Finally, the fact that the program was set at the general practice allowed a continuity of care.

Our results indicate that the rate of resolution achieved by nurses in 16 different minor illnesses is high (62.5\%), with low probability of return to consultation for the same reason (below 5\%). Interestingly, case resolution during the second year was higher compared to that of the first year, indicating that greater experience with the management algorithms resulted in an improved case resolution. We observed marked differences in the rate of case resolution between different conditions. For example, case resolution for burns and skin injury were much higher (over 90\%) than those for lower urinary symptoms and upper respiratory symptoms, which did not reach $50 \%$. Not resolved cases were sent to the GP for urgent consultation. Differences between resolution rates of the various minor illnesses might be related to the fact that some conditions correspond to long-established nurse practice (i.e. skin injury, burns) while others do not (i.e. upper respiratory symptoms, lower urinary symptoms). The relatively low resolution rate for these latter conditions probably indicates the compliance with signs of alarm that prompted referral to the GP as well as a high sense of responsibility of nurses in not assuming too complex processes. The rates of effectiveness in case resolution observed in the current study are high and support an approach using management algorithms for acute minor illnesses in primary care.

A limitation of the study is that case resolution was defined whenever the treatment protocol could be completed and treatment prescribed without the need of referral of the patient to the GP. Therefore, the resolution of the symptoms was not confirmed directly with the patient. Direct confirmation with the patient is hardly possible in a "real life" scenario. Return to consultation to primary care, either with a nurse or GP, was very low, averaging only $4.6 \%$, which is keeping with the high rate of case resolution observed. It is important to note, however, that some patients could have requested a second consultation outside primary care and could have not been captured by the system. It is important to mention that patients' satisfaction was not assessed in the current study. Therefore, the degree of satisfaction of patients by having been visited by a nurse instead of a GP could not be estimated. Randomized studies have demonstrated that in patients requesting same day consultation in primary care, the degree of satisfaction is greater when patients are treated by nurses than when they are treated by GPs $[4,6,7,16]$. There are however other studies with discrepant findings [11]. Finally, we did not evaluate the impact of the current approach in terms of cost. Further research will be required to evaluate the cost-effectiveness of the approach presented in this study.

\section{Conclusions}

In conclusion, the current study reports the results of a program of nurse algorithm-guided care for adult patients with acute minor illnesses requesting same day consultation to primary care based on close cooperation between nurses and GPs. The application of this program proved to be very effective in case resolution. The use of this type of programs may help nurses expand their role in primary care.

\section{Competing interests}

The authors declare that they have no competing interests.

\section{Authors' contributions}

NF conceived the study and participated in its design, analysis and interpretation of data, and writing of the manuscript. CS participated in the design of the study and analysis of data. EJ participated in the design of the study, analysis and interpretation of data, and helped to draft the manuscript. EA participated in the collection and analysis of data. DM participated in the collection and analysis of data. EC participated in the interpretation of data and helped to draft the manuscript. MU participated in the collection and analysis of data and helped to draft the manuscript. All authors have participated in the critical revision of the manuscript for important intellectual content and have read and approved the final manuscript.

\section{Acknowledgements}

The authors would like to acknowledge the work of RN and practitioners of all practices of the Institut Català de la Salut that participated in the care of patients reported in this study. The authors would also like to thank the work of Marco Pavesi in the statistical analysis of the results.

\section{Funding}

This research received no specific grant from any funding agency in the public, commercial, or not-for-profit sectors. 


\section{Author details}

'School of Nursing, University of Barcelona, Institut d'Investigació Biomèdica de Bellvitge (IDIBELL), Campus de Ciències de la Salut Bellvitge, Feixa Llarga s/n Pavello de Govern, 3a planta, 08907, L'Hospitalet de Llobregat, Catalunya, Spain. ${ }^{2}$ SAP Mataró, Institut Català de la Salut, Mataró, Catalunya, Spain. Institut Català de la Salut, Barcelona, Catalunya, Spain. ${ }^{4}$ EAP Premià, Institut Català de la Salut, Premià de Mar, Catalunya, Spain. ${ }^{5}$ EAP Pineda de Mar, Institut Català de la Salut, Pineda de Mar, Catalunya, Spain.

Received: 20 August 2012 Accepted: 8 May 2013

Published: 16 May 2013

\section{References}

1. Fairman JA, Rowe JW, Hassmiller S, Shalala DE: Broadening the scope of nursing practice. New Engl J Med 2011, 364:193-195.

2. The future of nursing: leading change, advancing health. Washington, DC: Institute of Medicine National Academies of Press; 2010.

3. High Quality Care for All: NHS Next Stage Review. London: Department of Health $\mathrm{DH} ; 2008$

4. Marsh GN, Dawes ML: Establishing a minor illness nurse in a busy general practice. Br Med J 1995, 310:778-780.

5. Myers PC, Lenci B, Sheldon MG: A nurse practitioner as the first point of contact for urgent medical problems in a general practice. Fam Pract 1997, 14:492-497.

6. Shum C, Humphreys A, Wheeler D, Cochrane MA, Skoda S, Clement S: Nurse management of patients with minor illnesses in general practice: multicentre randomized controlled trial. Br Med J 2000, 320:1038-1043.

7. Kinnerseley P, Anderson E, Parry K, Clement J, Archard L, Turton P. Randomised controlled trial of nurse practitioner versus general practitioner care for patients requesting "same day" consultations in primary care. Br Med J 2000, 320:1043-1048.

8. Horrocks S, Anderson E, Salisbury C: Systematic review of whether nurse practitioners working in primary care can provide equivalent care to doctors. Br Med J 2002, 324:819-23.

9. Laurant M, Reeves D, Hermens R, Braspenning J, Grol R, Sibbald B: Substitution of doctors by RN in primary care. Cochrane Database of Systematic 2004 Reviews 5:CD001271.

10. Lindblad E, Hallman EB, Gillsjö, Lindblad U, Fegerström L: Experiences of the new role of advanced practice RN in Swedish primary health care - A qualitative study. Int I Nurs Pract 2010, 16:69-74.

11. Rashid C: Benefits and limitations of RN taking on aspects of the clinical role of doctors in primary care: integrative literature review. J Adv Nurs 2010, 66:1658-1670.

12. Fabrellas N, Vidal A, Amat G, Lejardi Y, Deulofeu MP, Buendia C: Nurse management of "same day" consultation for patients with minor illnesses: results of an extended programme in primary care in Catalonia. J Adv Nurs 2010, 67:1811-1816.

13. Barnes $\mathrm{H}$, Crumie A, Carlisle C, Pilling D: Patients' perceptions of "uncertainty" in nurse practitioner consultations. Br J Nurs 2004, 13:1350-1354.

14. Caldow J, Bond C, Campbell N, San Miguel F, Kiger A, Lee A: Treatment of minor illness in primary care: a national survey of patient satisfaction, attitudes, and preferences regarding a wider nursing role. Health Expect 2007, 10:30-45.

15. Salisbury C, Munro J: Walk-in centres in primary care: a review of the international literature. Br J Gen Pract 2002, 53:53-59.

16. Venning P, Durie A, Roland M, Roberts C, Leese B: Randomised controlled trial comparing cost effectiveness of GP and nurse practitioners in primary care. Br Med J 2000, 320:1048-1053.

doi:10.1186/1471-2296-14-61

Cite this article as: Fabrellas et al: A program of nurse algorithm-guided care for adult patients with acute minor illnesses in primary care. BMC Family Practice 2013 14:61.

\section{Submit your next manuscript to BioMed Central and take full advantage of:}

- Convenient online submission

- Thorough peer review

- No space constraints or color figure charges

- Immediate publication on acceptance

- Inclusion in PubMed, CAS, Scopus and Google Scholar

- Research which is freely available for redistribution

Submit your manuscript at www.biomedcentral.com/submit
C Biomed Central 